\title{
Distinct Cytoplasmic Regions of the Human Granulocyte Colony-Stimulating Factor Receptor Involved in Induction of Proliferation and Maturation
}

\author{
FAN DONG, ${ }^{1,2}$ CARIN VAN BUITENEN,${ }^{1}$ KARIN POUWELS, ${ }^{1}$ LIES H. HOEFSLOOT, ${ }^{1}$ \\ BOB LÖWENBERG, ${ }^{1,2}$ AND IVO P. TOUW ${ }^{1 *}$ \\ Department of Hematology, Dr. Daniel den Hoed Cancer Center, P.O. Box 5201, 3008 AE Rotterdam, ${ }^{1}$ and \\ Institute of Hematology, Erasmus University, 3000 DR Rotterdam, ${ }^{2}$ The Netherlands
}

Received 15 July 1993/Returned for modification 23 August 1993/Accepted 14 September 1993

\begin{abstract}
The granulocyte colony-stimulating factor receptor (G-CSF-R) transduces signals important for the proliferation and maturation of myeloid progenitor cells. To identify functionally important regions in the cytoplasmic domain of the G-CSF-R, we compared the actions of the wild-type receptor, two mutants, and a natural splice variant in transfectants of the mouse pro-B cell line BAF3 and two myeloid cell lines, 32D and L-GM. A region of 55 amino acids adjacent to the transmembrane domain was found to be sufficient for generating a growth signal. The immediate downstream sequence of 30 amino acids substantially enhanced the growth signaling in the three cell lines. In contrast, the carboxy-terminal part of 98 amino acids strongly inhibited growth signaling in the two myeloid cell lines but not in BAF3 cells. Truncation of this region lead to an inability of the G-CSF-R to transduce maturation signals in L-GM cells. An alternative carboxy tail present in a splice variant of the G-CSF-R also inhibited growth signaling, notably in both the myeloid cells and BAF3 cells, but appeared not to be involved in maturation.
\end{abstract}

The formation of blood cells in the bone marrow is controlled by a regulatory network of hematopoietic growth factors $(5,23)$. One of these factors, granulocyte colonystimulating factor (G-CSF) secreted by macrophages, fibroblasts, and endothelial cells, plays an essential role in the regulation of granulopoiesis and the maintenance of neutrophil levels in the peripheral blood. For instance, G-CSF not only is able to stimulate the proliferation of neutrophilic progenitor cells but also induces maturation of these progenitor cells towards neutrophilic granulocytes $(8,24,32)$.

The murine and human receptors for G-CSF (G-CSF-R) consist of a single polypeptide with a molecular weight of 130,000 to $150,000(10,44)$. The high-affinity interaction between G-CSF and its receptor and subsequent activation of receptor signaling require the formation of homodimeric or oligomeric receptor complexes (10). The molecular cloning of the CDNAs encoding the murine and human G-CSF-R revealed that the murine and human G-CSF-R contain 812 and 813 amino acids, respectively, with a single transmembrane domain $(12,13,21)$. The structure of the G-CSF-R shows significant homology to that of the interleukin-6 (IL-6) signal transducer gp130 $(16,38)$. Expression of the G-CSF-R cDNAs in murine hematopoietic cells leads to formation of high-affinity G-CSF binding sites on the cell surface and renders these cells responsive to G-CSF $(11,49)$. At least four different forms of the human G-CSF-R, resulting from alternative splicing of G-CSF receptor mRNA, have been cloned from human placenta and myeloid leukemia (U937) cells $(13,21)$.

On the basis of structural similarities, the G-CSF-R has been classified as a member of the superfamily of cytokine receptors $(1,6)$. The homologous structures shared by the members of the family consist of four conserved cysteine residues and a WSXWS motif in the extracellular region.

\footnotetext{
* Corresponding author.
}

The cytoplasmic domain of the family is less conserved, but certain sequence similarities have been reported among the receptors for IL-3 to IL-7, G-CSF, granulocyte macrophageCSF, erythropoietin (EPO), and the $\beta$ chains of IL-2 and IL-3 $(11,29)$. Several studies have delineated functionally important regions in the cytoplasmic domain. For instance, a membrane-proximal region of the EPO receptor (EPO-R), IL-6 signal transducer gp130, and the $\beta$ chains of IL-2 and IL-3 is essential for transmitting a growth signal $(7,15,29$, 39). In agreement with these findings, it has been established that the membrane-proximal region of the G-CSF- $R$ is indispensible for growth signal transduction $(11,48)$. The cytoplasmic tail of the EPO-R has been shown to down-modulate the mitogenic response to $\operatorname{EPO}(7,35)$. In contrast, the distal cytoplasmic region of the G-CSF-R has been reported to enhance growth signaling potential (11).

In this paper, we describe the identification of two cytoplasmic regions of the wild-type (WT) G-CSF-R, located distal to the box 1- and box 2-containing region, one that positively and one that negatively regulates the mitogenic response to G-CSF. We provide evidence showing that a distinct carboxy terminus present in a G-CSF-R splice variant also inhibits growth signaling. Further, we show that the negative regulatory region of the WT G-CSF-R but not of the splice variant is essential for G-CSF-induced neutrophilic maturation. These findings establish that distinct regions of the cytoplasmic domain of the G-CSF-R have a determinative role in G-CSF-induced proliferation and maturation of myeloid progenitor cells.

\section{MATERIALS AND METHODS}

Cell lines and culture. The murine pro-B cell line BAF3 (34) was kindly provided by G. Plaetinck (Roche Research, Ghent, Belgium) and maintained in RPMI 1640 medium supplemented with $10 \%$ fetal calf serum (FCS) and $10 \mathrm{ng}$ of murine IL-3 per ml. Murine myeloid cell lines 32D (14) and 
L-GM (22) were provided by J. S. Greenberger (University of Massachusetts) and T. Honjo (Kyoto University Faculty of Medicine, Kyoto, Japan). 32D and L-GM cells were maintained in RPMI 1640 medium supplemented with $10 \%$ FCS and 10\% WEHI-3B cell conditioned medium (WEHI$\mathrm{CM}$ ) as a source of murine IL-3.

G-CSF-R expression constructs. For the construction of pLNCX-WT, the WT human G-CSF-R cDNA (13) (pHQ3, kindly provided by $S$. Nagata and R. Fukunaga) was excised from the pBluescript vector and inserted into the HpaI site of the retroviral expression vector pLNCX (25). For the construction of pLNCX-DC, reverse transcriptase-polymerase chain reaction (RT-PCR) was performed on total RNA isolated from normal granulocytes by using forward primer 5'TGTGATCATCGTGACTCCCTT3' (FW2) and reverse primer 5'CAAGATCTAGTTTACAATACTGAAG3' (RV2), and an 864-bp PCR fragment was subcloned into the pBluescript vector. A 248-bp stretch of the fragment was then cut out with BsrFI and ClaI and, together with the HindIII-BsrFI fragment obtained from pLNCX-WT, was inserted into HindIII-ClaI-cleaved pLNCX vector by triple ligation. Mutant DA was isolated from granulocytes of a patient with congenital neutropenia by RT-PCR using the same set of primers. The construction of the full-length DA mutant is essentially same as that described for the DC receptor. Mutant M1 was generated by PCR by introducing a termination codon at amino acid position 686 of the WT G-CSF-R. The PCR fragment was first ligated to the HincII site of the pBluescript vector and then excised from the plasmid by HindIII and XhoI digestion, blunt ended, and inserted into the HpaI site of pLNCX.

DNA transfection. The pLNCX expression constructs were linearized by $P v u I$ digestion and transfected into BAF3, 32D, and L-GM cells by electroporation. Following gene transfer, cells were cultured in IL-3-containing medium for $48 \mathrm{~h}$ and then selected in G418 (Gibco-BRL, Breda, The Netherlands)-containing medium at concentrations of 1.5 (BAF3) or 0.8 (32D and L-GM) mg/ml. G418-resistant clones were expanded and tested for their capacities to specifically bind radiolabeled G-CSF. RT-PCR using primer sets FW2RV1 (5'GTAGATCTTAGTCATGGGCTTATGG3'), FW2RV2, and FW2-RV3 (5'TCTCAGGGCACCGGCTTCTT TT3') was performed on the transfectants to test for possible incomplete transfer of sequences encoding the cytoplasmic domains.

G-CSF binding assay. Recombinant human G-CSF (Amgen, Thousand Oaks, Calif.) was radioiodinated by the method of Bolton and Hunter (2). G-CSF binding characteristics were determined as previously described (4). In brief, cells $\left(10^{6}\right)$ were incubated with serial dilutions of radiolabeled G-CSF ranging from $20 \mathrm{pM}$ to $5 \mathrm{nM}$ at $37^{\circ} \mathrm{C}$ for $1 \mathrm{~h}$. Incubations in the presence of excess nonlabeled G-CSF were also performed to correct for nonspecific binding. Bound ligand was separated from free ligand by centrifugation $(5 \mathrm{~min}$ at $1,000 \times g)$ through an ice-cold FCS layer. Receptor numbers and binding affinity were calculated as described previously (4).

DNA synthesis and cell proliferation assays. DNA synthesis was assayed by $\left[{ }^{3} \mathrm{H}\right]$ thymidine $\left(\left[{ }^{3} \mathrm{H}\right] \mathrm{TdR}\right)$ uptake. Cells $\left(10^{4}\right)$ were incubated in triplicate in $100-\mu$ l of $10 \%$ FCS-RPMI medium supplemented with titrated concentrations of G-CSF or WEHI-CM $(20 \%)$ in 96-well plates for $24 \mathrm{~h}$. Twelve hours before cell harvest, $1 \mu \mathrm{Ci}$ of $\left[{ }^{3} \mathrm{H}\right] \mathrm{TdR}(2$ $\mathrm{Ci} / \mathrm{mM}$; Amersham) was added to each well. $\left[{ }^{3} \mathrm{H}\right] \mathrm{TdR}$ incorporation was measured by liquid scintillation counting as described previously (40).
To quantify cell proliferation in suspension culture, cells were incubated in 25-ml culture flasks at an initial density of $0.5 \times 10^{5} / \mathrm{ml}(5 \mathrm{ml}$ of culture) in medium supplemented with recombinant human G-CSF at the indicated concentrations or without growth factors. Culture medium was refreshed every 2 to 3 days, and the cell densities were adjusted to approximately $0.5 \times 10^{5}$ to $1 \times 10^{5} / \mathrm{ml}$. Viable cells were counted on the basis of trypan blue exclusion.

Morphological examination. Cells were collected every 2 to 3 days and spun onto glass slides. Cell morphology was examined after May-Grünwald-Giemsa staining.

\section{RESULTS}

Properties of the G-CSF-R variant and mutants. The structures of the different forms of the human G-CSF-R relevant to this study are summarized in Fig. 1. DC represents an RT-PCR product obtained from normal granulocytes that is weakly expressed in comparison with the WT G-CSF-R (data not shown). Nucleotide sequencing revealed that $\mathrm{DC}$ is identical to the previously described splice variant D7, derived from placenta (21). From amino acid 727, DC is translated in an altered reading frame and contains 34 carboxy-terminal amino acids distinct from those of the WT G-CSF-R. DA, derived from granulocytes of a patient with congenital neutropenia, contains a C-to-T substitution at nucleotide position 2384 that introduces a TAG stop codon resulting in the truncation of 98 carboxy-terminal amino acids. No other alterations were found in this mutant by nucleotide sequencing. M1 contains only 55 amino acids in the cytoplasmic domain that includes the two subdomains called box 1 and box $2(11,29)$, with 3 additional amino acids downstream. The different forms of the G-CSF-R were introduced into the murine cell lines BAF3, 32D, and L-GM by electroporation. RT-PCR products derived from the transfectants by using primers that amplify the entire cytoplasmic domains all had the predicted sizes, indicating that no deletions in these regions had occurred during transfection and clone selection (Fig. 2).

G-CSF binding to BAF3, 32D, and L-GM transfectants. The numbers and dissociation constants of the G-CSF-R expressed by the different transfected cell lines were established following binding of ${ }^{125}$ I-radiolabeled G-CSF (Table 1). While the parental BAF3, 32D, and L-GM cells lacked specific G-CSF binding (data not shown), the different BAF3 transfectants (WT, DC, DA, and M1) expressed G-CSF binding sites with affinities ranging from 0.28 to $2.04 \mathrm{nM}$ (Table 1), i.e., comparable to those of the G-CSF-R expressed on human peripheral blood neutrophils (4) and those of the G-CSF-R expressed on cell line transfectants $(13,21$, 49). 32D/WT, 32D/DC, 32D/DA, and L-GM/M1 transfectants expressed G-CSF-R with similar average numbers and affinities. On the other hand, 32D/M1, L-GM/WT, L-GM/ DC, and L-GM/DA transfectants expressed G-CSF-R at detectable but lower densities (less than 100 sites per cell), which did not allow estimation of binding affinities (Table 1). The reasons for these variations in surface expression levels of the G-CSF-R are unknown, but they may relate to differences in protein stability or transportation in the different cell line transfectants.

Mitogenic signaling by difierent forms of G-CSF-R. The abilities of different forms of the G-CSF-R to transduce a proliferative signal were first examined in $\left[{ }^{3} \mathrm{H}\right] \mathrm{TdR}$ uptake assays. At least three independent clones of each construct were examined, giving comparable results. Parental BAF3, 32D, and L-GM cells or G418-resistant clones transfected 

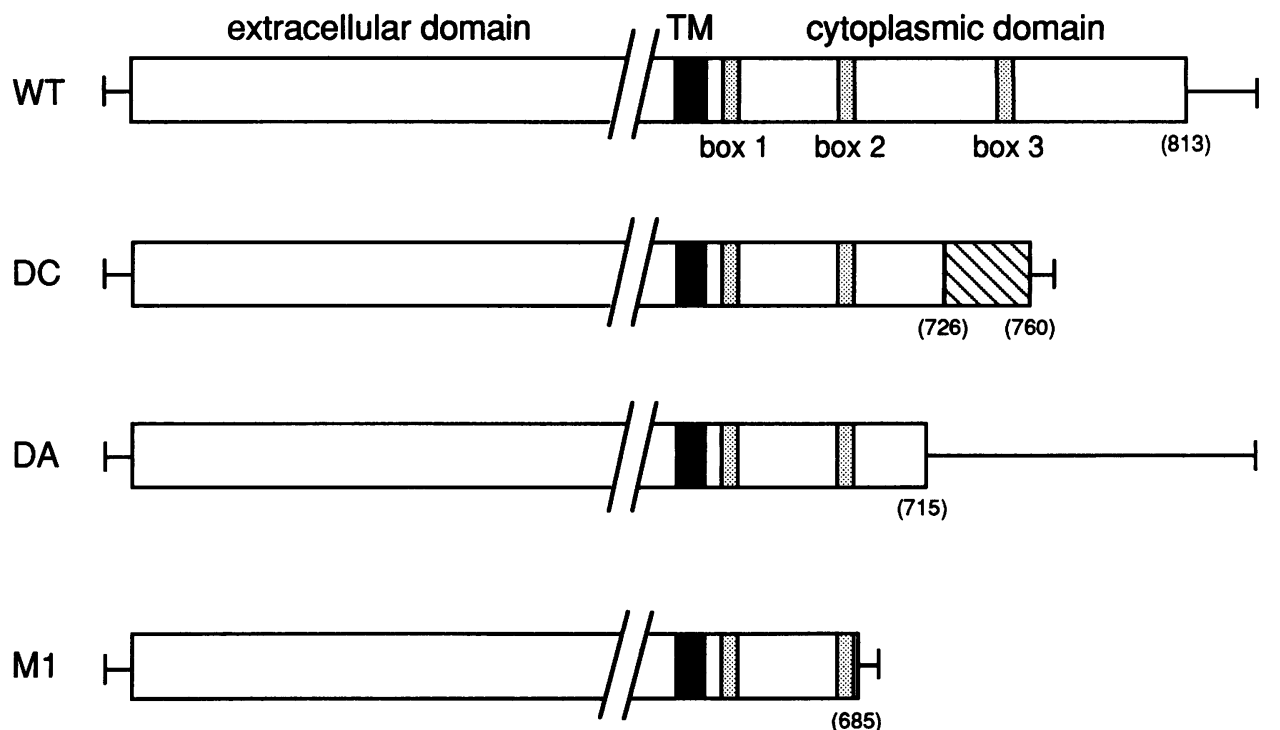

FIG. 1. Schematic diagram of the G-CSF-R cDNAs. Boxes 1,2, and 3 denote subdomains conserved in some members of the cytokine receptor superfamily. The hatched box indicates the distinct cytoplasmic tail of the DC receptor from the corresponding region of the WT receptor (see text). Lines indicate noncoding regions. The numbers in parentheses mark amino acid positions. TM, transmembrane domain.

with the vector without G-CSF-R showed no response to G-CSF (Table 2). G-CSF induced DNA synthesis in BAF3 cells expressing the WT G-CSF-R to a level of approximately 40 to $50 \%$ of that induced by the optimal concentration of WEHI-CM $(20 \%)$ as a source of murine IL-3 (Table 2 and Fig. 3A, BAF/WT). Deletion of 98 amino acids from the carboxy terminus did not negatively affect G-CSF-induced growth signaling in BAF3 cells (Table 2 and Fig. 3A, BAF/DA). However, further deletion of 30 amino acids significantly reduced the G-CSF sensitivity, resulting in a 100 -fold increase in the G-CSF concentration $(>100 \mathrm{ng} / \mathrm{ml})$ required to elicit a maximal response (Fig. 3A, BAF/M1). BAF3 cells expressing the DC receptor, which contains 11 additional amino acids as compared with the DA receptor, followed by a distinct carboxy tail, also displayed a reduced responsiveness: the magnitude of maximal response was about 30 to $50 \%$ of that of BAF/WT cells (Table 2 and Fig. 3A, BAF/DC).

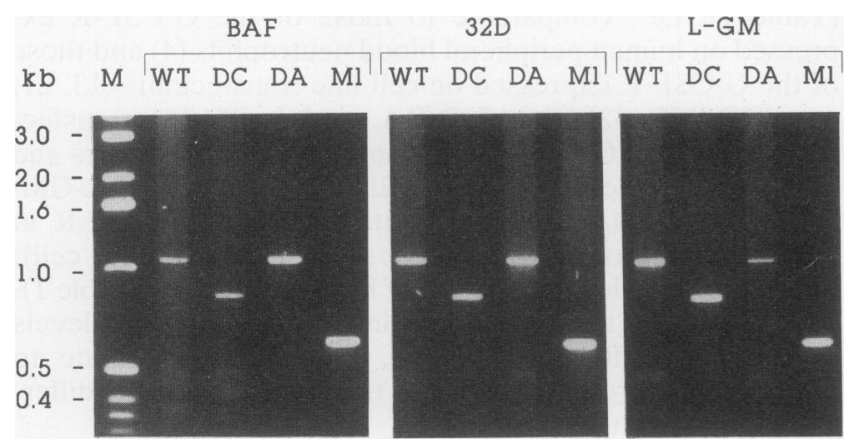

FIG. 2. RT-PCR analysis of the G-CSF-R cytoplasmic domain in BAF3, 32D, and L-GM transfectants. Primer sets used for amplification were FW2-RV1 for WT and DA, FW2-RV2 for DC, and FW2-RV3 for M1. For further details, see Materials and Methods. Expected sizes of PCR products: 1,106 bp for WT and DA, 864 bp for DC, and $630 \mathrm{bp}$ for M1.
In contrast to the BAF transfectants, 32D cells expressing the WT G-CSF-R exhibited only a weak mitogenic response to G-CSF (Table 2 and Fig. 3B, 32D/WT). Similar responses to G-CSF were observed in 32D cells expressing the DC or the M1 receptor (Table 2 and Fig. 3B). The 32D/DA cells, however, showed a dramatic increase in responsiveness to G-CSF (Fig. 3B, 32D/DA). Additional titration experiments revealed that $32 \mathrm{D} / \mathrm{DA}$ cells proliferated maximally at $0.1 \mathrm{ng}$ of G-CSF per ml (data not shown).

L-GM cells transfected with the different forms of the G-CSF-R displayed a response pattern similar to that of 32D transfectants (Table 2 and Fig. 3C). L-GM cells expressing the WT G-CSF receptor showed a poor mitogenic response to G-CSF, as did L-GM subclones expressing the DC or the

TABLE 1. Binding properties of the G-CSF receptors on cell line transfectants ${ }^{a}$

\begin{tabular}{lcc}
\hline Cell type & $\begin{array}{c}\text { No. of } \\
\text { sites/cell }\end{array}$ & $K_{d}(\mathrm{nM})$ \\
\hline BAF/WT & 3,030 & 2.04 \\
BAF/DC & 1,500 & 0.67 \\
BAF/DA & 2,560 & 0.28 \\
BAF/M1 & 31,410 & 0.60 \\
32D/WT & 1,640 & \\
32D/DC & 750 & 1.86 \\
32D/DA & 2,630 & 0.68 \\
32D/M1 & $+^{b}$ & 0.53 \\
& & ND $^{c}$ \\
L-GM/WT & + & ND \\
L-GM/DC & + & ND \\
L-GM/DA & + & ND \\
L-GM/M1 & 1,290 & 1.84 \\
\hline
\end{tabular}

${ }^{a}$ Parental BAF3, 32D, and L-GM cells did not show specific G-CSF binding.

$b+$, specific binding detectable but too low for an accurate estimation of receptor numbers and affinities.

$c \mathrm{ND}$, not determined. 
TABLE 2. Mitogenic responses of cell line transfectants in $\left[{ }^{3} \mathrm{H}\right] \mathrm{TdR}$ uptake assay after $48 \mathrm{~h}$ of culture

\begin{tabular}{lccc}
\hline & \multicolumn{3}{c}{ dpm (mean \pm SD) with: } \\
\cline { 2 - 4 } Transfectant & No factor & G-CSF $(10 \mathrm{ng} / \mathrm{ml})$ & $\begin{array}{c}\text { IL-3 }(20 \% \\
\text { WEHI-CM) }\end{array}$ \\
\hline BAF/Neo & $33 \pm 6$ & $52 \pm 20$ & $46,298 \pm 463$ \\
BAF/WT & $35 \pm 14$ & $17,845 \pm 393$ & $36,833 \pm 1,289$ \\
BAF/DA & $45 \pm 6$ & $16,390 \pm 344$ & $41,772 \pm 1,128$ \\
BAF/DC & $20 \pm 4$ & $5,485 \pm 527$ & $19,673 \pm 156$ \\
BAF/M1 & $17 \pm 5$ & $7,190 \pm 360$ & $31,021 \pm 434$ \\
& & & \\
32D/Neo & $33 \pm 13$ & $29 \pm 20$ & $24,452 \pm 196$ \\
32D/WT & $53 \pm 21$ & $954 \pm 134$ & $19,780 \pm 692$ \\
32D/DA & $76 \pm 47$ & $22,606 \pm 633$ & $18,312 \pm 549$ \\
32D/DC & $38 \pm 7$ & $1,256 \pm 72$ & $14,765 \pm 221$ \\
32D/M1 & $149 \pm 52$ & $1,309 \pm 94$ & $27,554 \pm 1,350$ \\
& & & \\
L-GM/Neo & $40 \pm 13$ & $39 \pm 8$ & $2,968 \pm 297$ \\
L-GM/WT & $29 \pm 7$ & $383 \pm 61$ & $3,183 \pm 267$ \\
L-GM/DA & $43 \pm 5$ & $5,317 \pm 393$ & $3,293 \pm 59$ \\
L-GM/DC & $22 \pm 4$ & $441 \pm 46$ & $1,566 \pm 49$ \\
L-GM/M1 & $22 \pm 5$ & $560 \pm 50$ & $1,369 \pm 178$ \\
\hline
\end{tabular}

M1 receptor. L-GM/DA cells, on the other hand, showed a high rate of DNA synthesis even at low concentrations $(0.1$ $\mathrm{ng} / \mathrm{ml}$ ) of G-CSF.

Cell proliferation of transfectants. We next examined whether G-CSF could replace IL-3 as a stimulus for sustained proliferation of transfectants. As shown in Fig. 4A and B, BAF/WT and BAF/DA cells became G-CSF dependent and grew equally well in 1 and $100 \mathrm{ng}$ of G-CSF per ml. Although BAF/DC and BAF/M1 cells could also grow continuously at $100 \mathrm{ng}$ of G-CSF per ml with a reduced rate of proliferation (Fig. 4B), these cells could not survive at $1 \mathrm{ng}$ of G-CSF per ml (Fig. 4A). Cells proliferating exponentially on G-CSF could be propagated for several months. The response of $\mathrm{BAF} / \mathrm{M} 1$ cells indicates that the membraneproximal region of 55 amino acids is sufficient for generating a growth signal. In addition, the data show that a region between amino acids 56 and 85 in the cytoplasmic domain enhances growth signaling. The C-terminal region of the DC receptor, which contains an additional 11 amino acids of the WT receptor and 34 different amino acids in comparison with the DA mutant (Fig. 1), exerted a negative effect on growth signaling in BAF3 cells.

The 32D transfectants expressing the WT, DC, and M1 receptors died 4 days after transfer to G-CSF-containing medium (Fig. 4C). In contrast, 32D/DA cells proliferated continuously upon stimulation with G-CSF (Fig. 4C). Similarly, L-GM/DA cells could be cultured continuously in G-CSF-containing medium, whereas L-GM cells expressing the WT, DC, or M1 receptor gradually lost viability (Fig. 4D). Thus, the characteristics of the responses observed in 32D and L-GM subclones define a functional subdomain located in the distal carboxy-terminal region of the WT G-CSF-R that appears to suppress the growth signaling in myeloid cells. The altered carboxy terminus of the DC receptor also inhibits G-CSF-induced proliferation of the two myeloid cell lines.

Induction of myeloid maturation by difierent forms of G-CSF-R. A prominent feature of G-CSF is its capacity to induce neutrophilic differentiation. 32Dc13 and L-G cells, variant cell lines of 32D and L-GM cells, respectively, have previously been shown to mature into neutrophilic granulocytes in response to G-CSF $(22,45)$. We therefore examined whether L-GM and 32D cells expressing the different G-CSF receptor structures would mature towards neutrophilic granulocytes following G-CSF induction. L-GM/WT cells exhibited immature myeloblastic features when cultured in IL-3containing medium (Fig. 5a). Upon transfer to G-CSFcontaining medium, these cells gradually developed into mature neutrophilic granulocytes, showing an enlarged cytoplasm-to-nucleus ratio and lobulated nuclei. On day 9 of G-CSF induction, all cells displayed a morphology characteristic of mature neutrophils (Fig. 5b), similar to that of mature L-G cells (22). As shown in Fig. 5c and d, L-GM/DC and L-GM/DA cells did not mature towards granulocytes in G-CSF-containing medium. Identical results were obtained with L-GM/M1 transfectants (data not shown). Addition of different concentrations of murine granulocyte macrophageCSF or murine IL-3 to the G-CSF-containing culture medium to prolong cell survival also failed to induce terminal maturation of L-GM/DC or L-GM/M1 cells (data not shown).

32D/WT cells, which were myeloblastic when cultured with IL-3 (Fig. 5e), did not mature upon transfer to G-CSFcontaining medium. Instead, these cells manifested a pheno-
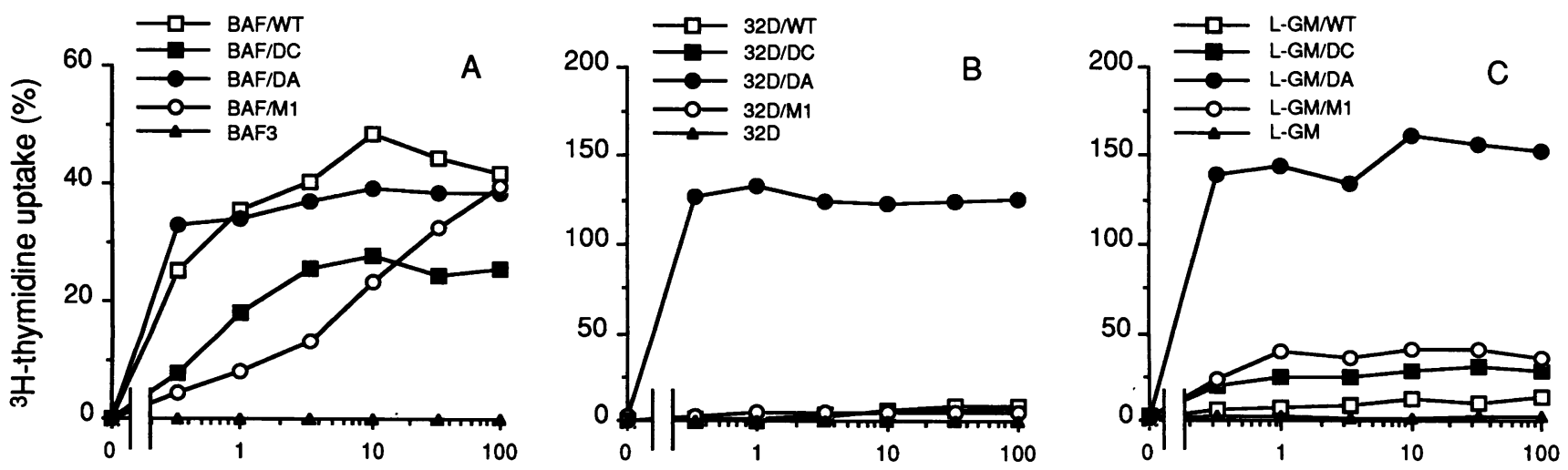

Recombinant human G-CSF (ng/ml)

FIG. 3. G-CSF responsiveness of BAF3, 32D, and L-GM cells expressing different forms of the G-CSF-R. Cell proliferation was measured by the $\left[{ }^{3} \mathrm{H}\right] \mathrm{TdR}$ uptake assay. Data represent the percentage of maximal response to IL-3 (20\% WEHI-CM). 

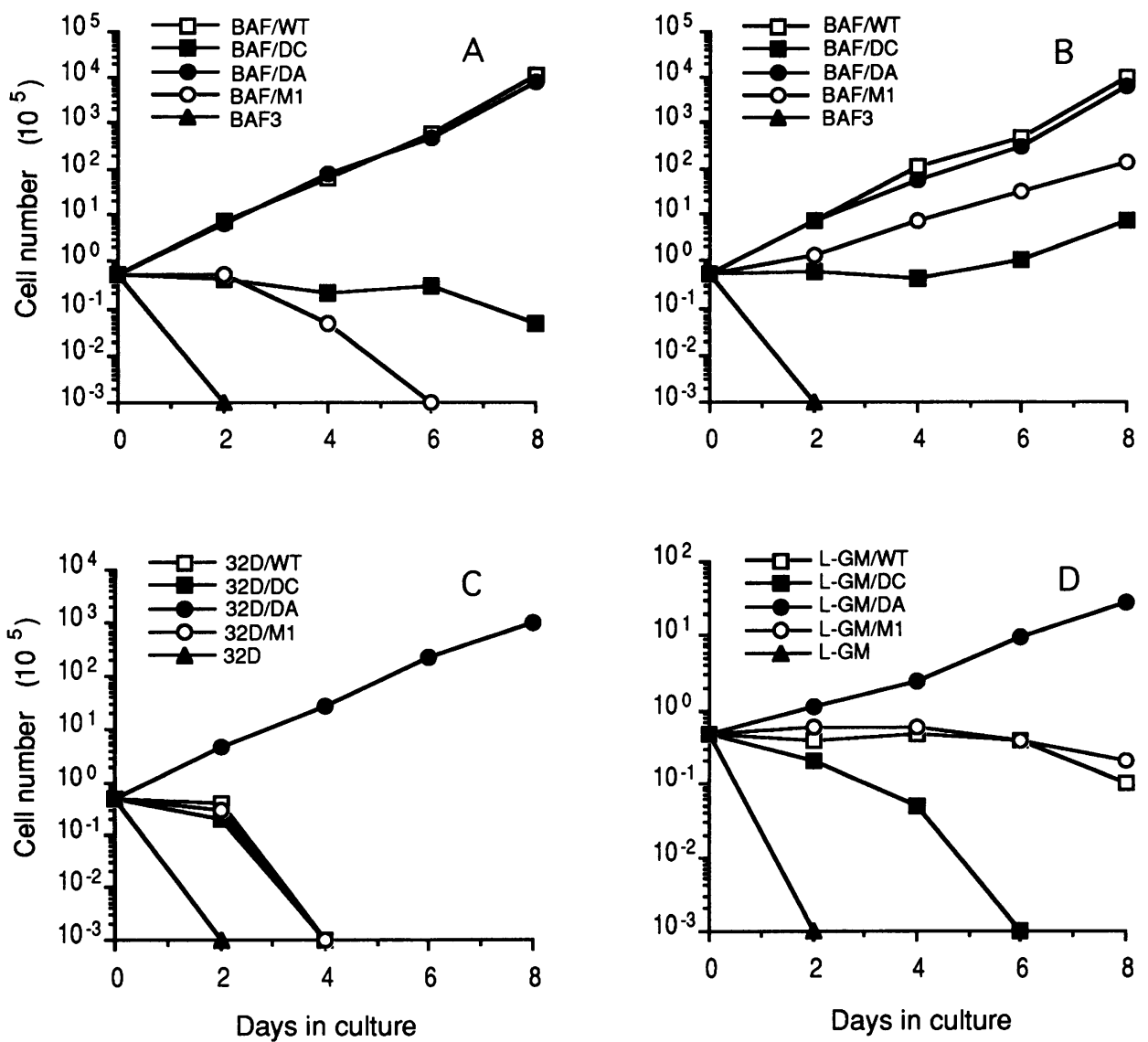

FIG. 4. G-CSF-dependent long-term growth of BAF3, 32D, and L-GM transfectants. Cells maintained in IL-3-containing medium were washed extensively and transferred to G-CSF-containing medium. Viable cell counts were determined at the indicated times. BAF3 subclones were cultured in medium containing 1 (A) and 100 (B) ng of G-CSF per ml. 32D (C) and L-GM (D) transfectants were cultured in $10 \mathrm{ng}$ of G-CSF per ml, except for DA subclones (1 $\mathrm{ng}$ of G-CSF per ml).

type typical of apoptosis (i.e., condensation of nuclei) after culture in G-CSF-containing medium for 4 days (Fig. 5f). To exclude the possibility that these cells died from a lack of growth signaling before they could undergo myeloid maturation, cells were grown in G-CSF-containing medium supplemented with different concentrations of WEHI-CM (1, $2.5,5$, and $10 \%$ ) to allow survival for up to 2 weeks, but still no maturation was noted (data not shown). Similarly, $32 \mathrm{D} / \mathrm{DC}$ or $32 \mathrm{D} / \mathrm{M} 1$ subclones were rapidly lost in cultures supplemented with G-CSF and failed to mature (Fig. $5 \mathrm{~g}$ and data not shown). 32D/DA cells also showed no evidence of terminal myeloid maturation when maintained in G-CSF (Fig. 5h). These results show that the 32D cells used in this study were incapable of granulocytic maturation in response to G-CSF, suggesting a defect in the G-CSF-induced maturation signaling pathway in these cells.

\section{DISCUSSION}

In this study, we have delineated distinct functional regions in the cytoplasmic domain of the human G-CSF-R. We show here that a membrane-proximal region of 55 amino acids of the human G-CSF-R is sufficient to generate a mitogenic signal, consistent with a recent report by Ziegler et al. (48). This region contains two subdomains, termed box 1 and box 2, which are well conserved among some members of the cytokine receptor superfamily $(11,29)$. The impor- tance of the box 1- and box 2-containing region for growth signal transduction has been demonstrated for several cytokine receptors $(7,15,26,29,35,39,48)$. It is likely that this region is involved in activation of common or highly related signal transduction pathways. This notion is supported by several receptor transfection studies $(7,15,29,35,48)$ and by the fact that some of the ligands for these receptors induce similar patterns of tyrosine phosphorylation $(9,27$, 36).

The most prominent finding of this study is that the 98 carboxy-terminal amino acids of the WT G-CSF-R appeared to be involved in neutrophilic maturation. Little is known of the mechanisms by which hematopoietic growth factors control maturation. For instance, it has remained a matter of discussion whether activation of growth factor receptors truly drives maturation or simply supports the proliferation and survival of cells already programmed to mature along a certain hematopoietic lineage $(18,28,33)$. With regard to the G-CSF-R, our results with the L-GM transfectants clearly favor the first possibility, because L-GM/WT cells developed into morphologically mature granulocytes when cultured in G-CSF, whereas L-GM/DA cells proliferated and survived in G-CSF without any evidence of maturation. This failure of L-GM/DA cells to mature was thus apparent in spite of their ability to proliferate in response to G-CSF. L-GM/DC and L-GM/M1 cells, which proliferated modestly 


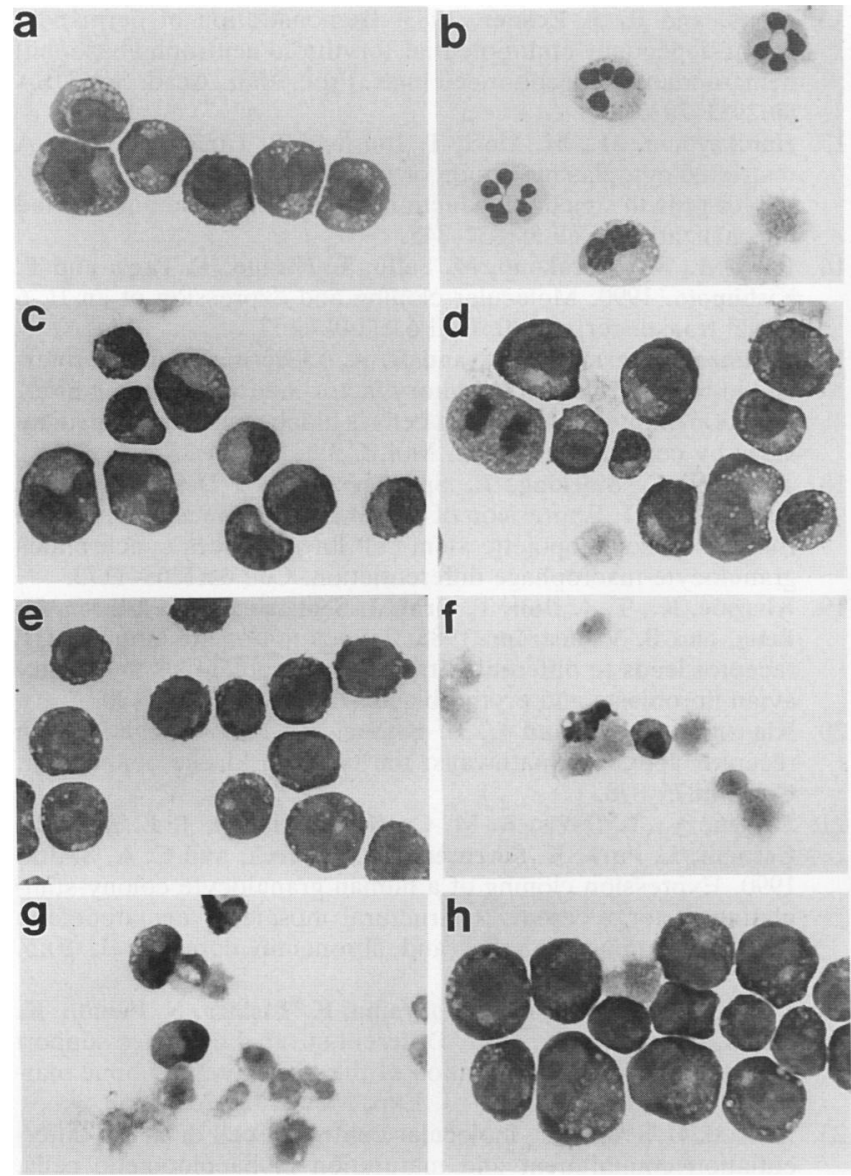

FIG. 5. Morphological analysis of 32D and L-GM transfectants. Shown are L-GM (a) and 32D (e) cells maintained in IL-3-containing medium (10\% WEHI-CM); L-GM/WT (b), L-GM/DC (c), and L-GM/DA (d) cells cultured in medium containing $10 \mathrm{ng}$ of G-CSF per $\mathrm{ml}$; and 32D/WT (f), 32D/DC (g), and 32/DA (h) cells in G-CSF-containing medium. May-Grünwald-Giemsa staining was used (original magnification, $\times 63$ ).

in response to G-CSF, were also incapable of maturation. Our data therefore indicate that the carboxy-terminal part of the WT G-CSF-R contains a subdomain critical for maturation signaling that is required neither for proliferation nor for survival. A linkage between the carboxy-terminal truncation of the IL-2 gamma chain and T-cell maturation arrest in patients with $X$-linked severe combined immunodeficiency has recently been suggested (30). In G-CSF-R, a subdomain designated box 3 has been identified in this region, which is also present in the IL-6 signal transducer gp130 (11). Notably, IL-6 also stimulates granulocytic colony formation by normal mouse marrow cells (42) and was able to induce maturation of M1 leukemia cells $(17,41)$. Moreover, leukemia inhibitory factor and oncostatin $\mathrm{M}$, which share gp130 for signal transduction, have also been reported to induce maturation of M1 cells (3). Thus, it appears likely that the box 3 subdomain may have a direct role in the induction of maturation, possibly through association with a specific cytoplasmic substrate.

The presence of the carboxy-terminal region in the WT G-CSF-R strongly inhibits the mitogenic signaling potential of the G-CSF-R in two myeloid cell lines, 32D and L-GM cells (Fig. 3). In contrast, this negative effect on growth signaling was not evident in pro-B BAF3 cells, in which the WT G-CSF-R transduces a growth signal as strong as that of the DA mutant. Despite their enhanced sensitivity to G-CSF, 32D/DA and L-GM/DA cells remained fully factor dependent and died within $48 \mathrm{~h}$ upon withdrawal of IL-3 or G-CSF (data not shown).

In contrast to the L-GM transfectants, 32D cells expressing the WT G-CSF-R did not mature upon exposure to G-CSF. However, the cells responded only transiently to G-CSF in proliferation assays and could not be maintained in G-CSF-containing suspension culture. These findings suggest that the negative effect of the carboxy terminus of the WT G-CSF-R on proliferation and the induction of terminal maturation may be uncoupled and raise the possibility that separate domains within this region are involved in these functions. Alternatively, this could be explained by a lack of downstream molecules involved in the maturation signaling in the $32 \mathrm{D}$ cells.

It is still unclear by which mechanism proliferation signaling in myeloid cells is inhibited by the carboxy-terminal region of the WT G-CSF-R. Protein phosphorylation on tyrosine or serine/threonine residues has been shown to be frequently involved in the regulation of receptor function, both positively and negatively $(20,43)$. One possibility is that the down-regulation of the activity of the G-CSF-R is caused by receptor phosphorylation, as has been demonstrated for EPO-R (46). It is of note that, in contrast to our results with BAF3/WT transfectants, the negative regulatory region of the EPO-R also acted in BAF3 cells, suggesting that the carboxy-terminal inhibitory regions of the G-CSF-R and EPO-R act through different mechanisms. Alternatively, this region may contain a subdomain that specifically activates downstream molecules which transmit growth-inhibitory signals. In that case, these molecules would not be active in BAF3 cells. A negative effect of the carboxy-terminal sequence on growth signal transduction has been reported for several other growth factor receptors, including those of the receptor tyrosine kinase family $(7,19,37,47)$. Thus, negative regulation of proliferation signaling by the carboxyterminal parts of receptors may be seen as a common feature of different growth factor receptor systems.

We have further shown that a region of 30 amino acids between residues 56 and 85 markedly augments the mitogenic signaling potential of the G-CSF-R. A similar region has been identified in the EPO-R and in the common $\beta$ chain of IL-3, granulocyte macrophage-CSF, and IL-5 receptor $(31,39)$. It remains to be established whether this region is actively involved in transduction of a mitogenic signal or is important simply for a proper conformation that facilitates signaling from the box 1- and box 2-containing membraneproximal regions. In this respect, it is relevant that Ziegler et al. have demonstrated that the region between amino acids 57 and 96 in the cytoplasmic domain of the G-CSF-R is required for the induction of acute-phase protein gene expression by G-CSF in hepatoma cell line transfectants (48). These data appear to suggest that this region indeed contains a functional subdomain.

Whether the DC splice variant has a physiological role in hematopoiesis is as yet unknown. We have shown that the DC variant receptor is able to transduce a mitogenic signal in the three murine cell lines, consistent with a previous report (49). However, the growth signal transduced by the DC receptor is significantly weaker than that transduced by the DA mutant, suggesting that the alternative carboxy tail also acts as a down-modulator of proliferation. Unlike the nega- 
tive regulatory region of the WT G-CSF-R, the DC tail portion exerts its effect in both lymphoid and myeloid cells, indicating that it must act by a mechanism different from that of the WT G-CSF-R. Because the amino acid sequence in this portion is rather hydrophobic (21), one possibility is that the alternative tail folds towards the cell membrane, thereby hindering the association of growth-signaling molecules with other regions. Because the DC receptor is incompetent for maturation induction, it might act as a negative regulator of maturation at certain stages of myeloid development.

Our findings may have implications for studies dealing with the granulocytic maturation defects in certain hematological diseases, such as neutropenias and acute myeloid leukemia. The fact that G-CSF-R mutant DA was isolated from a patient with severe congenital neutropenia strongly suggests that defective G-CSF-R signaling had been involved in the course of the disease, a possibility that is currently being examined in detail.

\section{ACKNOWLEDGMENTS}

We thank H. Hoogerbrugge for radiolabeling of G-CSF, R. Delwel for critical reading of the manuscript, and $\mathrm{K}$. de Groot for assistance with figure preparation.

This work was supported by the Dutch Cancer Society.

\section{REFERENCES}

1. Bazan, J. F. 1989. A novel family of growth factor receptors: a common binding domain in the growth hormone, prolactin, erythropoietin and IL-6 receptor, and the p75 IL-2 receptor beta chain. Biochem. Biophys. Res. Commun. 164:788-793.

2. Bolton, A. E., and W. M. Hunter. 1973. The labelling of proteins to high specific radioactivities by conjugation to a ${ }^{125}$ I-containing acylating agent. Biochem. J. 133:529-539.

3. Bruce, A. G., I. H. Hoggatt, and T. M. Rose. 1992. Oncostatin M is a differentiation factor for myeloid leukemia cells. J. Immunol. 149:1271-1275.

4. Budel, L. M., I. P. Touw, R. Delwel, and B. Löwenberg. 1989. Granulocyte colony-stimulating factor receptors in human acute myelocytic leukemia. Blood 74:2668-2673.

5. Clark, S. C., and R. Kamen. 1987. The human hematopoietic colony-stimulating factors. Science 236:1229-1237.

6. Cosman, D., S. D. Lyman, R. L. Idzerda, M. P. Beckmann, L. S. Park, R. G. Goodwin, and C. J. March. 1990. A new cytokine receptor superfamily. Trends. Biosci. 15:265-270.

7. D'Andrea, A. D., A. Yoshimura, H. Youssoufian, L. I. Zon, J.-W. Koo, and H. Lodish. 1991. The cytoplasmic region of the erythropoietin receptor contains nonoverlapping positive and negative growth-regulatory domains. Mol. Cell. Biol. 11:1980 1987.

8. Demetri, G. D., and J. D. Griffin. 1991. Granulocyte colonystimulating factor and its receptor. Blood 78:2791-2808.

9. Evans, J. P. M., A. R. Mire-Sluis, A. V. Hofibrand, and R. G. Wickremasinghe. 1990. Binding of G-CSF, GM-CSF, tumor necrosis factor- $\alpha$, and gamma-interferon to cell surface receptors on human myeloid leukemia cells triggers rapid tyrosine and serine phosphorylation of a 75-Kd protein. Blood 75:88-95.

10. Fukunaga, R., E. Ishizaka-Ikeda, and S. Nagata. 1990. Purification and characterization of the receptor for murine granulocyte colony-stimulating factor. J. Biol. Chem. 265:14008-14015.

11. Fukunaga, R., E. Ishizaka-Ikeda, C.-X. Pan, Y. Seto, and S. Nagata. 1991. Functional domains of the granulocyte colonystimulating factor receptor. EMBO J. 10:2855-2865.

12. Fukunaga, R., E. Ishizaka-Ikeda, Y. Seto, and S. Nagata. 1990. Expression cloning of a receptor for murine granulocyte colonystimulating factor. Cell 61:341-350.

13. Fukunaga, R., Y. Seto, S. Mizushima, and S. Nagata. 1990. Three different mRNAs encoding human granulocyte colonystimulating factor receptor. Proc. Natl. Acad. Sci. USA 87: 8702-8706.

14. Greenberger, J. S., M. A. Sakakeeny, R. K. Humphries, C. J.
Eaves, and R. J. Eckner. 1983. Demonstration of permanent factor-dependent multipotential (erythroid/neutrophil/basophil) hematopoietic progenitor cell lines. Proc. Natl. Acad. Sci. USA 80:2931-2935.

15. Hatakeyama, M., M. Mori, T. Doi, and T. Taniguchi. 1989. A restricted cytoplasmic region of IL-2 receptor $\beta$ chain is essential for growth signal transduction but not for ligand binding and internalization. Cell 59:837-845.

16. Hibi, M., M. Murakami, M. Saito, T. Hirano, T. Taga, and T. Kishimoto. 1990. Molecular cloning and expression of an IL-6 signal transducer, gp130. Cell 63:1149-1157.

17. Hofiman-Liebermann, B., and D. A. Liebermann. 1991. Interleukin-6- and leukemia inhibitory factor-induced terminal differentiation of myeloid leukemia cells is blocked at an intermediate stage by constitutive c-myc. Mol. Cell. Biol. 11:2375-2381.

18. Just, U., C. Stocking, E. Spooncer, T. M. Dexter, and W. Ostertag. 1991. Expression of the GM-CSF gene after retroviral transfer in hematopoietic stem cell lines induces synchronous granulocyte-macrophage differentiation. Cell 64:1163-1173.

19. Khazaie, K., T. J. Dull, T. Graf, J. Schlessinger, A. Ullrich, H. Beug, and B. Vennström. 1988. Truncation of the human EGF receptor leads to differential transforming potentials in primary avian fibroblasts and erythroblasts. EMBO J. 7:3061-3071.

20. Klausner, R. D., and L. E. Samuelson. 1991. T cell antigen receptor activation pathways: the tyrosine kinase connection. Cell 64:875-878.

21. Larsen, A., T. Davis, B. M. Curtis, S. Gimpel, J. E. Sims, D. Cosman, L. Park, E. Sorensen, C. J. March, and C. A. Smith. 1990. Expression cloning of a human granulocyte colony-stimulating factor receptor: a structural mosaic of hematopoietin receptor, immunoglobulin, and fibronectin domains. J. Exp. Med. 172:1559-1570.

22. Lee, K. H., T. Kinashi, K. Tohyama, K. Tashiro, N. Funato, K. Hama, and T. Honjo. 1991. Different stromal cell lines support lineage-selective differentiation of the multipotential bone marrow stem cell clone LyD9. J. Exp. Med. 173:1257-1266.

23. Metcalf, D. 1989. The molecular control of cell division, differentiation commitment and maturation in haemopoietic cells. Nature (London) 339:27-30.

24. Metcalf, D. 1991. Control of granulocytes and macrophages: molecular, cellular, and clinical aspects. Science 254:529-533.

25. Miller, A. D., and G. J. Rosman. 1989. Improved retroviral vectors for gene transfer and expression. BioTechniques 7:980 990.

26. Miura, O., J. L. Cleveland, and J. N. Ihle. 1993. Inactivation of erythropoietin receptor function by point mutations in a region having homology with other cytokine receptors. Mol. Cell. Biol. 13:1788-1795.

27. Miura, O., A. D'Andrea, D. Kabat, and J. N. Ihle. 1991. Induction of tyrosine phosphorylation by the erythropoietin receptor correlates with mitogenesis. Mol. Cell. Biol. 11:48954902.

28. Miyajima, A., T. Kitamura, N. Harada, T. Yokota, and K. Arai. 1992. Cytokine receptors and signal transduction. Annu. Rev. Immunol. 10:295-331.

29. Murakami, M., M. Narazaki, M. Hibi, H. Yawata, K. Yasukawa, M. Hamaguchi, T. Taga, and T. Kishimoto. 1991. Critical cytoplasmic region of the interleukin 6 signal transducer gp130 is conserved in the cytokine receptor family. Proc. Natl. Acad. Sci. USA 88:11349-11353.

30. Naguchi, M., H. Yi, H. M. Rosenblatt, A. H. Filipovich, S. Adelstein, W. S. Modi, O. W. McBride, and W. J. Leonard. 1993. Interleukin-2 receptor gamma chain mutation results in $\mathrm{X}$-linked combined immunodeficiency in humans. Cell 73:147157.

31. Nakamura, Y., N. Komatsu, and H. Nakauchi. 1992. A truncated erythropoietin receptor that fails to prevent programmed cell death of erythroid cells. Science 257:1138-1141.

32. Nicola, N. A. 1987. Granulocyte colony-stimulating factor and differentiation-induction in myeloid leukemia cells. Int. J. Cell Cloning 5:1-15.

33. Ogawa, M. 1993. Differentiation and proliferation of hematopoietic stem cells. Blood 81:2844-2853. 
34. Palacios, R., and M. Steinmetz. 1985. IL-3-dependent mouse clones that express B-220 surface antigen, contain Ig genes in germ-line configuration, and generate $B$ lymphocytes in vivo. Cell 41:727-734.

35. Quelle, D. E., and D. M. Wojchowski. 1991. Localized cytosolic domains of the erythropoietin receptor regulate growth signaling and down-modulate responsiveness to granulocyte-macrophage colony-stimulating factor. Proc. Natl. Acad. Sci. USA 88:48014805.

36. Quelle, F. W., D. E. Quelle, and D. M. Wojchowski. 1992. Interleukin 3, granulocyte-macrophage colony-stimulating factor, and transfected erythropoietin receptors mediate tyrosine phosphorylation of a common cytosolic protein (pp100) in FDC-ER cells. J. Biol. Chem. 267:17055-17060.

37. Roussel, M. F., T. J. Dull, C. W. Rettenmier, P. Ralph, A. Ullrich, and C. J. Sherr. 1987. Transforming potential of the c-fms proto-oncogene (CSF-1 receptor). Nature (London) 325: 549-552.

38. Saito, M., K. Yoshida, M. Hibi, T. Taga, and T. Kishimoto. 1992. Molecular cloning of a murine IL-6 receptor-associated signal transducer, gp130, and its regulated expression in vivo. J. Immunol. 148:4066-4071.

39. Sakamaki, K., I. Miyajima, T. Kitamura, and A. Miyajima. 1992. Critical cytoplasmic domain of the common $\beta$ subunit of the human GM-CSF, IL-3 and IL-5 receptors for growth signal transduction and tyrosine phosphorylation. EMBO J. 11:35413549.

40. Salem, M., R. Delwel, I. Touw, L. Mahmoud, and B. Löwenberg. 1988. Human AML colony growth in serum-free culture. Leuk. Res. 12:157-165.

41. Selvakumaran, M., D. A. Liebermann, and B. Hoffman-Liebermann. 1992. Deregulated c-myb disrupts interleukin-6- or leukemia inhibitory factor-induced myeloid differentiation prior to c-myc: role in leukemogenesis. Mol. Cell. Biol. 12:2493-2500.

42. Suda, T., Y. Yamaguchi, J. Suda, Y. Miura, A. Okano, and Y. Akiyama. 1988. Effect of interleukin 6 (IL-6) on the differentiation and proliferation of murine and human hemopoietic progenitors. Exp. Hematol. 16:891-895.

43. Ullrich, A., and J. Schlessinger. 1990. Signal transduction by receptors with tyrosine kinase activity. Cell 61:203-212.

44. Uzumaki, H., T. Okabe, N. Sasaki, K. Hagiwara, F. Takaku, and S. Itoh. 1988. Characterization of receptor for granulocyte colony-stimulating factor on human circulating neutrophils. Biochem. Biophys. Res. Commun. 156:1026-1032.

45. Valtieri, M., D. J. Tweardy, D. Caracciolo, K. Johnson, F. Mavilio, S. Altmann, D. Santoli, and G. Rovera. 1987. Cytokinedependent granulocytic differentiation: regulation of proliferative and differentiative responses in a murine progenitor cell line. J. Immunol. 138:3829-3835.

46. Yoshimura, A., and H. F. Lodish. 1992. In vitro phosphorylation of the erythropoietin receptor and an associated protein, pp130. Mol. Cell. Biol. 12:706-715.

47. Yoshimura, A., G. Longmore, and H. F. Lodish. 1990. Point mutation in the exoplasmic domain of the erythropoietin receptor resulting in hormone-independent activation and tumorigenicity. Nature (London) 348:647-649.

48. Ziegler, S. F., T. A. Bird, K. K. Morella, B. Mosley, D. P. Gearing, and H. Baumann. 1993. Distinct regions of the human granulocyte-colony-stimulating factor receptor cytoplasmic domain are required for proliferation and gene induction. Mol. Cell. Biol. 13:2384-2390.

49. Ziegler, S. F., T. Davis, J. A. Schneringer, T. L. Franklin, T. W. Tough, M. Teepe, A. Larsen, D. E. Williams, and C. A. Smith. 1991. Alternative forms of the human G-CSF receptor functions in growth signal transduction. New Biol. 3:1242-1248. 